\title{
Tazobactam/piperacillin for moderate-to-severe pneumonia in patients with risk for aspiration: comparison with imipenem/cilastatin.
}

\section{$\operatorname{AUTHOR}(\mathrm{S}):$}

Ito, Isao; Kadowaki, Seizo; Tanabe, Naoya; Haruna, Akane; Kase, Masahito; Yasutomo, Yoshiro; Tsukino, Mitsuhiro; ... Chin, Kazuo; Ichiyama, Satoshi; Mishima, Michiaki

\section{CITATION:}

Ito, Isao ...[et al]. Tazobactam/piperacillin for moderate-to-severe pneumonia in patients with risk for aspiration: comparison with imipenem/cilastatin.. Pulmonary pharmacology \& therapeutics 2010, 23(5): 403-410

\section{ISSUE DATE:}

2010-10

\section{URL:}

http://hdl.handle.net/2433/126728

\section{RIGHT:}

(C) 2010 Elsevier Ltd; This is not the published version. Please cite only the published version.; この論文は出版社版でありません。引用の際に は出版社版をご確認ご利用ください。 
Tazobactam/piperacillin for moderate-to-severe pneumonia in patients with risk for aspiration: comparison with imipenem/cilastatin

Isao Ito $^{\text {a, }}{ }^{*}$, Seizo Kadowaki ${ }^{b}$, Naoya Tanabe ${ }^{\mathrm{a}}$, Akane Haruna ${ }^{\mathrm{a}}$, Masahito Kase ${ }^{\mathrm{b}}$, Yoshiro Yasutomo ${ }^{\mathrm{b}}$, Mitsuhiro Tsukino $^{\mathrm{c}}$, Asako Nakai $^{\mathrm{d}}$, Hisako Matsumoto ${ }^{\mathrm{a}}$, Akio Niimi $^{\mathrm{a}}$, Kazuo Chin ${ }^{\mathrm{e}}$, Satoshi Ichiyama ${ }^{\mathrm{f}}$, Michiaki Mishima ${ }^{\mathrm{a}}$

${ }^{\mathrm{a}}$ Department of Respiratory Medicine, ${ }^{\mathrm{d}}$ Department of Diagnostic Imaging and Nuclear Medicine, ${ }^{\mathrm{e}}$ Department of Respiratory Care and Sleep Medicine, ${ }^{\mathrm{f}}$ Department of Infecton Control and Prevention, Kyoto University Hospital, 54

Shogoin-kawaracho, Sakyo, Kyoto, Japan

${ }^{\mathrm{b}}$ Department of Medicine, Ono Municipal Hospital, 323 Naka-cho, Ono, Hyogo, Japan

${ }^{c}$ Department of Respiratory Medicine, Hikone Municipal Hospital, 1822 Yasaka-cho, Hikone, Shiga, Japan

*Correspondence to: Isao Ito, $\mathrm{MD}, \mathrm{PhD}$.

Department of Respiratory Medicine, Kyoto University Hospital,

54 Shogoin-kawaharacho, Sakyo, Kyoto, 606-8507, Japan.

Email: isaoito@kuhp.kyoto-u.ac.jp

Tel: +81757513884

Fax: +81757514643 


\section{Abstract}

Background: Treatment of aspiration pneumonia is becoming an important issue due to aging of populations worldwide. Effectiveness of tazobactam/piperacillin (TAZ/PIPC) in aspiration pneumonia is not clear.

Purpose: To compare clinical efficacy between TAZ/PIPC (1:4 compound) and imipenem/cilastatin (IPM/CS) in patients with moderate-to-severe aspiration pneumonia.

Patients and methods: In this open-label, randomized study either TAZ/PIPC $5 \mathrm{~g}$ or IPM/CS $1 \mathrm{~g}$ was intravenously administered every $12 \mathrm{hr}$ to patients with moderate-to-severe community-acquired aspiration pneumonia or nursing-home acquired pneumonia with risk for aspiration pneumonia for average 11 days. The primary outcome was clinical response rate at the end of treatment (EOT) in validated per-protocol (VPP) population. Secondary outcomes were clinical response during treatment (days 4 and 7) and at the end of study (EOS) in VPP population, and survival at day 30 in modified intention-to-treat (MITT) population.

Results: There was no difference between the groups in primary or secondary outcome. However, significantly faster improvement as measured by axillary temperature $(p<0.05)$ and WBC count $(p=0.01)$ was observed under TAZ/PIPC treatment. In patients with gram-positive bacterial infection, TAZ/PIPC was more effective at EOT in VPP population $(p=0.03)$.

Conclusion: TAZ/PIPC is as effective and safe as IPM/CS in the treatment of moderate- to-severe aspiration pneumonia. 
Keywords: Community-acquired pneumonia (CAP); Nursing home-acquired pneumonia (NHAP); Aspiration; Tazobactam/piperacillin (TAZ/PIPC);

Imipenem/cilastain (IPM/CS); Open-label randomized study 


\section{Introduction}

Although societies are aging across the developed world [1], Japan's population in particular is rapidly graying more than that seen elsewhere, such that elderly individuals account for $20.8 \%$ of the total population. According to statistics provided by the World Health Organization (WHO) in 2004, lower respiratory tract infections are the third most common cause of death worldwide [2] and are ranked fourth in Japan [3]. More than $90 \%$ of deaths due to pneumonia occur in elderly persons aged $>65$ years, and the disease claims the highest mortality among the senior elderly aged $>85$ years. For this reason, the importance of treating elderly patients with pneumonia is growing as the world population ages.

In elderly people, one of the most common forms of pneumonia is aspiration pneumonia due to a decrease or disturbance in the function of swallowing. The elderly often exhibit physiologically decreased swallowing and cough reflexes; micro-aspiration of oral bacteria or upper respiratory tract secretion is repeated asymptomatically during nocturnal sleep [4]. Bacteriologically, pathogenic organisms of aspiration pneumonia include Streptococcus pneumonia (S. pneumoniae), Haemophilus influenzae, Staphylococcus aureus, Streptococcus milleri group, microaerophils, and anaerobic bacteria [5-8]. It is also known that in the elderly populations mixed infection with several pathogens including S. pneumoniae and beta-lactamase-producing bacteria such as $H$. influenzae frequently occurs $[9,10]$.

Tazobactam/piperacillin (TAZ/PIPC) is widely used for the treatment of this entity, because it is stable to beta-lactamases and effective against both gram-positive and gram-negative bacteria. Broad-spectrum carbapenem antibiotics are often used for the treatment of pneumonia in the elderly and have been shown to be effective against aspiration pneumonia [11-13]. On the other hand, in cases of Pseudomonas 
aeruginosa ( $P$. aeruginosa) infection, carbapenem antibiotics have a higher risk of developing resistant bacteria than penicillin antibiotics $[14,15]$. Because patients at risk for aspiration have many occasions to use antibiotics, there is concern that in these individuals frequent use of carbapenems may lead to an increase in resistant bacteria.

From a clinical point of view, pneumonia in the elderly population is often severe, difficult to treat, and accompanied by various complications. Indeed, increased age in community-acquired pneumonia (CAP) is related to increased mortality and the severity assessment of CAP is adjusted for age in the Pneumonia Severity Index (PSI) and CURB-65 pneumonia severity score $[16,17,18]$. However, only a few prospective studies have evaluated the therapeutic effects of antibiotics in aspiration pneumonia $[11,19,20]$.

In Japan, although TAZ/PIPC at a ratio of 1:4, instead of 1:8, has been successfully used for the treatment of severe infections such as sepsis, its effectiveness against CAP or aspiration pneumonia has not yet been elucidated. TAZ/PIPC exhibits almost the same antibacterial spectrum as carbapenem antibiotics. Thus, whether TAZ/PIPC could be an alternative therapeutic option in moderate-to-severe aspiration pneumonia is important information, considering the risk of developing resistant bacteria by frequent use of carbapenems. In this report, we compare the clinical effectiveness and safety of TAZ/PIPC with that of IPM/CS in the treatment of moderate-to-severe aspiration pneumonia. 


\section{Materials and methods}

\subsection{Patients}

Patients aged $\geq 15$ years with a risk for aspiration who had been hospitalized after developing moderate-to-severe pneumonia in the community or nursing home were enrolled. Pneumonia was diagnosed by radiological findings of a new and/or progressive infiltrate(s) and $\geq 2$ of the following conditions: cough, sputum or change of sputum character (increased volume and/or purulence), dyspnea, tachypnea, abnormal breathing sound, pleuritic chest pain, auscultatory findings on chest examination consistent with the lung infiltrate, documented axillary body temperature $\geq 37.5^{\circ} \mathrm{C}$ within the past $24 \mathrm{~h}$, rigors and/or chills, general malaise, and WBC count $<3000 / \mathrm{mm}^{3}$ or $\geq 10,000 / \mathrm{mm}^{3}$. Severity of pneumonia was assessed by PSI [12]; those with severity class IV-V were enrolled. Patients were judged to be at risk for aspiration if they had $\geq 1$ of the following conditions: neurological disorder such as cerebrovascular diseases, neuromuscular diseases, and dementia, bedridden state, oral/pharyngeal/throat disorder, gastroesophageal disorder such as esophageal diverticulum, achalasia, systemic sclerosis, esophageal cancer, GERD, post-gastrectomy (total or partial), and hiatal hernia, usage of sedatives or hypnotics, insertion of a nasogastric tube, subjective or observed aspiration/choking/dysphagia, and episode of vomiting [21].

Patients with any of the following were excluded: hospital-acquired pneumonia, hospitalization within 60 days prior to development of symptom(s), immunocompromising disease or receipt of immunocompromising therapy, active lung cancer, terminal illness, pregnancy or breastfeeding, known allergy to the indicated antibiotics, presence of other infiltrative diseases such as radiation pneumonitis, organizing pneumonia, drug-induced pneumonia, and obstructive 
pneumonia, tuberculosis or fungal infection, and empyema.

\subsection{Setting and design}

This prospective, single center, open-label, randomized, comparative study was conducted from June 2003 to May 2007 at Ono Municipal Hospital. The study was approved by the institutional review board and written informed consent was obtained from all patients. Following enrollment, the patients were randomly assigned to receive either imipenem/cilastatin (IPM/CS 1:1) $1 \mathrm{~g}$ or TAZ/PIPC (1:4) $5 \mathrm{~g}$ given intravenously every $12 \mathrm{~h}$ for $7-14$ days, until defervescence $\left(<37^{\circ} \mathrm{C}\right)$ for $48 \mathrm{~h}$ with clinical stability without worsening of dyspnea, sputum or level of C-reactive protein. If recurrence of fever $\left(>37.5^{\circ} \mathrm{C}\right)$ was observed during antibiotic treatment in recovering patients, the therapy was continued for 4 days from the day of recurrent fever. The reason for the setting of twice-daily regimen was that most of the patients with risk for aspiration pneumonia were expected to be elderly and their age-related decreased renal function was taken in consideration. For patients with decreased documented and/or calculated creatinine clearance rate (Ccr) on admission, the q 12-h dose of TAZ/PIPC or IPM/CS was adjusted as follows: Ccr 10-50 mL/min, $2.5 \mathrm{~g}$ or 0.25 g every $12 \mathrm{~h}$, respectively; Ccr $<10 \mathrm{~mL} / \mathrm{min}, 1.25 \mathrm{~g}$ or $0.125 \mathrm{~g}$, respectively. In patients with PSI class V, intravenous erythromycin $500 \mathrm{mg}$ every $12 \mathrm{~h}$ was added. Use of other add-on antibiotics was not permitted.

\subsection{Clinical and bacteriologic evaluation}

Baseline assessments included PSI scores (including nursing-home residency), risk for aspiration, comorbid illness, immunosuppressive treatment, prior antibiotic treatment, and allergy to antibiotics. Clinical signs and symptoms (axillary 
temperature, respiratory rate, oxygen saturation, volume and character of sputum, and degree of dyspnea and malaise), chest radiography, and laboratory tests (complete blood count, serum chemistry, and C-reactive protein) were evaluated before treatment (admission day 1), during treatment (days 4 and 7), and at the end of treatment (EOT; days 7-14). At the end of study (EOS; day 28-35), late response was evaluated.

Microbiological examinations were performed as described previously [22]. Before initiating treatment with antibiotics, sputum samples were collected for Gram's stain and cultures where possible. Blood samples were obtained for culture. Urine samples were obtained and tested for urinary antigens of S. pneumoniae (Binax NOW S.pneumoniae urinary antigen test; Inverness Medical Innovations, Waltham, MA, USA) and Legionella pneumophila serogroup 1 (Binax NOW Legionella urinary antigen test). For serological examinations, Mycoplasma pneumoniae antibody was tested by paired particle hemagglutinin test and that of Chlamydophila pneumoniae by paired ELISA (Hitazyme; Hitachi Chemical, Tokyo, Japan). Antigen tests for influenza virus A and B were performed using throat or nasal swab samples between November and March.

\subsection{Criteria of evaluation}

Because substantial numbers of patients were expected to present with recurrence of fever due to aspiration in the period between EOT and EOS, the primary efficacy variable was defined as the clinical response in validated per-protocol (VPP) population at EOT. The secondary efficacy variables were clinical response during treatment (days 4 and 7) and at EOS in VPP population and survival at day 30 in modified intention-to-treat (MITT) population. Patients in the VPP population had to 
receive treatment with the study $\operatorname{drug}(\mathrm{s})$ for $\geq 72 \mathrm{~h}$ in cases of clinical failure or 4 days in cases of clinical cure without protocol violation or missing data. Excluded from the VPP population were those who were lacking for information or clinical data, were treated with other antibiotics concomitantly with the study drug, or were treated with systemic corticosteroid resulting in interfering judgment of efficacy of study drugs. MITT population included all randomized patients who had received $\geq 1$ dose of study drug.

Clinical response was based on blinded investigators' global assessment of clinical signs and symptoms, chest radiography, WBC count, and serum CRP. Chest radiography and serum CRP levels were used for the assessment only on day 7 , because it is known that they might appear to worsen on day 4 when compared to day 1 even if the patient's condition is clinically improving. Clinical response was categorized as improving, no obvious change or indeterminate, or worsening. When judged in the latter 2 categories, the test drug was discontinued and an alternative antibiotic(s) was given. Late response at EOS was evaluated as follows: cure, resolution of signs and symptoms related to pneumonia; relapse, recurrent fever or aspiration pneumonia after initial improvement; failure, deterioration of signs and symptoms of pneumonia, lack of resolution, or need for alternative antibiotic(s) for pneumonia.

\subsection{Statistical analysis}

As for background factors and baseline laboratory data, continuous variables are indicated as the average values \pm standard deviations. Variations in evaluation items from the baseline and intergroup differences in measured values were assessed by Student's $t$-test, Wilcoxon signed-rank test, or Mann-Whitney $U$-test, whereas 
differences in population rate between the groups were evaluated by chi-square test.

The significance level was set at $<0.05$ for two-tailed test. 


\section{Results}

\subsection{Patients}

In the study period, 369 patients with CAP and 100 patients with nursing home-acquired pneumonia (NHAP) were treated at our hospital. Among the CAP patients, 193 had risk for aspiration, whereas all NHAP patients were judged at risk. Among the 293 patients with aspiration risk, 212 were classified as PSI of IV-V. Finally, 163 patients who fulfilled the criteria for MITT population were enrolled in this study (Fig. 1). Eighty-one patients were assigned to receive TAZ/PIPC and 82 patients to IPM/CS. Eight patients did not fulfill inclusion criteria (6 patients who did not receive the study drug for $\geq 72$ hrs and 2 who took i.v. corticosteroids); therefore excluding these individuals the VPP population comprised 76 patients on TAZ/PIPC and 79 on IPM/CS.

Baseline demographic and clinical characteristics for MITT population are shown in Table 1; baseline data were similar between the 2 groups. In MITT population, duration of therapy (mean $\pm \mathrm{SD}$ ) was $10.6 \pm 4.2$ days in TAZ/PIPC group and $11.1 \pm 4.6$ days in IPM/CS group $(p=0.48)$.

\subsection{Clinical outcomes}

Primary and secondary outcomes are summarized in Table 2. At EOT, the clinical effective rate for VPP population in TAZ/PIPC and IPM/CS groups was $83 \%$ and $82 \%$, respectively ( $p=0.92$; Fig. 2 ). Time-course analysis on axillary temperatures, CRP, and WBC counts in MITT population are shown in Fig.3. Significant improvement was observed on day 4 compared to day 1 in all 3 parameters in both groups. However, axillary temperature and WBC counts were significantly lower in TAZ/PIPC group compared to IPM/CS group on day 4, showing more prompt improvement among 
patients on TAZ/PIPC. There were no significant differences between the groups in secondary outcome measures.

Mortality rate within 30 days of admission in MITT population was $15 \%$ on TAZ/PIPC group and 24\% on IPM/CS, which was not significantly different $(p=0.12)$. Major causes of death in the 2 groups were recurrence of pneumonia in 6 and 3, cardiac failure in 2 and 2, and sepsis in 1 and 2 patients in TAZ/PIPC and IPM/CS group, respectively.

\subsection{Bacteriologic analysis}

Microbiologic diagnosis was estimated in 84 out of 163 patients (52\%; Table 3). S.pneumoniae was detected in 23 patients (28\%) on TAZ/PIPC and 19 patients (23\%) on IPM/CS. Of these individuals, 4 patients in the TAZ/PIPC and 5 patients in the IPM/CS group were suggested to have mixed infection with other bacteria or nonbacterial pathogens. Although in patients with gram-positive bacterial infection there was no intergroup difference of efficacy at EOT as ascertained in the MITT population ( $p=0.11$ ), a significantly higher efficacy was noted in the TAZ/PIPC than IPM/CS group in the VPP population ( $p=0.03$; Table 4). In patients with gram-positive bacterial infection, body temperature ( $p<0.001$; Fig. 4A) and WBC count ( $p=0.02$; Fig. $4 \mathrm{~B})$ on day 4 were lower in the TAZ/PIPC group than in IPM/CS group. In patients with gram-negative bacterial infection or in patients without any pathogens identified, no intergroup difference was found in efficacy (Table 4 and 5).

\subsection{Safety and tolerability}

All VPP population were evaluated for safety. Adverse events probably related to study drug were recorded in 24 of 76 TAZ/PIPC recipients ( 24 events) and 30 of 80 
IPM/CS recipients (32 events; Table 6). The most frequent adverse event was diarrhea in both groups, affecting 21 patients (28\%) on TAZ/PIPC and 25 patients (31\%) on IPM/CS. Treatment was not interrupted in any patients due to adverse events. 


\section{Discussion}

In this study, TAZ/PIPC or IPM/CS was administered to moderate-to-severe pneumonia in patients at risk of aspiration for an average of 11 days and resulted in similar efficacy for both drugs and no intergroup difference in incidence of adverse events. On treatment day 4, fever subsided and CRP and WBC count significantly decreased in both treatment groups, although alleviation of fever and decrease of WBC count occurred more rapidly in the TAZ/PIPC group.

Pathogenic bacteria were detected in about $50 \%$ of our patients, and a half of these were attributed to gram-positive bacteria including S. pneumoniae. Although anaerobic bacteria are undetectable with routine sputum culture, it has been suggested that anaerobes, to which both TAZ/PIPC and IPM/CS are highly sensitive [23,24], play an important role in the development of aspiration pneumonia. According to the Infectious Diseases Society of America (IDSA)/the American Thoracic Society (ATS) guideline [16], TAZ/PIPC is recommended as drug of choice for the treatment of aspiration pneumonia and CAP due to Pseudomonas spp. or anaerobic bacteria. From a bacteriological point of view, it is suggested that TAZ/PIPC is as useful as IPM/CS in the treatment of aspiration pneumonia due to anaerobic bacteria, gram-positive bacteria, or gram-negative bacillary bacteria.

In our study, compared to IPM/CS, TAZ/PIPC more rapidly improved fever and $\mathrm{WBC}$ count on day 4 in patients with gram-positive bacterial infection. It has previously been reported that, compared to ceftazidime plus amikacin, TAZ/PIPC plus amikacin exerted significantly rapider antipyretic action in cancer patients with granulocytopenia [25]. Since elderly patients affected with pneumonia often experience impaired activities of daily living (ADLs) even after pneumonia has cleared, such rapid antipyretic action may be favorable for improving ADLs in these 
individuals and promoting early ambulation.

In the treatment of elderly aspiration pneumonia, it is sometimes difficult to decide when to stop treatment with antibiotics because recurrent fever is often observed in this population. Unless signs suggestive of worsening of pneumonia or of occurrence of another pneumonia are observed, it is reasonable to refrain from prolonged treatment with wide spectrum antibiotics. Another difficulty in treating such patients is that they sometimes present with lung abscess. In the current study, we found 5 patients (5\%) with abscess out of 97 patients screened with CT scan (data not shown). Only one of the 5 required antibiotic treatment for more than 14 days, which suggests that treatment longer than 14 days is not required for non-cavitating $\underline{\text { lung abscess. }}$

In this study, P. aeruginosa was isolated from only a few patients. This finding supports previous reports suggesting that, in Japan, only $1 \%$ of NHAP patients [26] and 6\% of those with healthcare-associated pneumonia (HCAP) patients [27] had P. aeruginosa as pathogenic bacterium, whereas this organism was isolated from about $25 \%$ of patients with NHAP or HCAP in Europe and the United States [28,29]. In Japan, $P$. aeruginosa is rarely found as pathogenic bacterium of NHAP probably because patients with serious underlying diseases such as chronic respiratory failure usually undergo treatment in hospitals rather than nursing homes. Monitoring of responsible pathogens in NHAP patients may be warranted on alert of possible increase of $P$. aeruginosa arising in this population.

In this study, we have a treated mixed populations, including both patients from nursing homes and not from nursing homes. The former are categorized as $\underline{\text { HCAP according to the ATS guidelines [30], and are recommended to be treated with }}$ anti-pseudomonal beta-lactam combined with either fluroquinolone or 
aminoglycoside, and anti-MRSA antibiotics. We launched this study before the publication of the guidelines and did not include anti-MRSA antibiotics, which may have resulted in the $20 \%$ of failure at EOT in each group. Indeed, resistant pathogens $\underline{\text { such as MRSA and Pseudomonas were isolated in } 8 \% \text { of the subgroup (data not }}$ shown). On the other hand, in 44 patients with neither HCAP nor hospitalization in the previous 5 years, resistant pathogens were isolated in only one patient ( $2 \%$, data not shown). Even in elderly patients at risk for aspiration, the wide spectrum antibiotics used in this study might not have been required. Further, we did not establish a de-escalation regimen in this study. As the ATS guidelines recommend [30], it would have been appropriate to apply de-escalation therapy when resistant pathogens were not isolated because the population of this study was at risk for repetitive aspiration pneumonias.

As mentioned above, treatment of HCAP including NHAP is recommended $\underline{\text { to include coverage for drug-resistant pathogens such as MRSA and P. aeruginosa }}$ [30]. So far, several studies have reported the same levels of efficacy and safety for $\underline{\text { TAZ/PIPC in the treatment of nosocomial infections including peritonitis [31] and }}$ $\underline{\text { HAP [31-33] as compared with IPM/CS, and HAP [34] and VAP [35] as compared }}$ with ceftazidime. Although most of HAPs can be attributed to aspiration, no study has examined the efficacy of TAZ/PIPC in the treatment of aspiration pneumonia in the elderly. Indeed, the mean age of our study population was 85 years, while patients in the previous studies were 52-67 years old [31-35]. It is suggested that carbapenem antibiotics have a higher risk of encouraging resistant bacteria than penicillin antibiotics $[19,20]$. Patients at risk of aspiration pneumonia often undergo repeated anti-pneumonia treatment. From the viewpoint of reducing carbapenem-resistant pathogens, it is therefore reasonable to use TAZ/PIPC as one drug of choice for the 
treatment of moderate-to-severe aspiration pneumonia.

In our cohort of elderly patients, the two test drugs were given twice daily in consideration of their probable age-related decrease of renal function. In view of the antibacterial activities [23,24] and pharmacokinetics [36,37] of TAZ/PIPC in response to the major pathogens of aspiration pneumonia such as Pneumococcus and oral anaerobic bacteria, it is possible to achieve the clinical utility of TAZ/PIPC ( $1 \mathrm{~g} / 4 \mathrm{~g})$ with twice-daily administration. The observed efficacy rate was as high as $83 \%$ at EOT with twice-daily administration presumably because $S$. pneumoniae and anaerobic bacteria, although the latter are undetected by culture, accounted for most of the bacterial infections in our study population. However, such an administration schedule of TAZ/PIPC every 6 hours should be attempted in populations in whom $P$. aeruginosa is isolated frequently [38-40], and this regimen would have been appropriate in this study as well. From the same point of view, the dose of IPM/CS may not have been sufficient, considering the usual adult dosage of 500mg every $6 \mathrm{~h}$ or $1 \mathrm{~g}$ every $8 \mathrm{~h}$ mentioned in the guidelines [30]. Thus, interpretation of the equal efficacy in the primary endpoint as well as the small difference in the early response in fever requires caution. Even though the observed efficacy in a short period was as high as $90 \%$ in both groups, the low dosages may have affected the decreased rates of $\underline{\text { cure at EOT. }}$

TAZ/PIPC used in this study was an injectable preparation containing a combination of tazobactam, a beta-lactamase inhibitor, and piperacillin, a broad-spectrum penicillin antibiotic, with a titer ratio of $1: 4$. It is reported that tazobactam and piperacillin exert the maximum antimicrobial activity when used with a titer ratio of 1:8 to 2:1 [41]. However, there remains a concern whether the ratio of $1: 4$ could have influenced the results. Since the combination ratio of $1: 8$ is widely 
available worldwide, further examination is required to determine whether the results of this study are applicable to TAZ/PIPC (1:8) preparations or not. 


\section{Conclusions}

TAZ/PIPC is as effective and safe as IPM/CS for the treatment of moderate-to-severe aspiration pneumonia, with faster recovery of fever. The most frequent adverse event was diarrhea in both treatment groups. Although the results should be interpreted with $\underline{\text { caution taking the low-dosage regimens into account, both drugs are potential }}$ treatment options in moderate-to-severe aspiration pneumonia in the elderly.

\section{Acknowledgment}

We greatly thank Drs Hiroyuki Namura, Hiroya Sakuramoto, Yoichiro Kusumoto, Masamichi Nasu, Akira Kawamura, Rei Ueno, Atsushi Kurohara, Norihito Shibata, Kenichi Kimura, and Keisho Chin at Ono Municipal Hospital for patients care and data collection. We thank Mr Hirofumi Okazaki and Ms Masako Fujiwara for their laboratory work.

\section{Conflict of interest}

None declared. 


\section{Figure captions}

Fig. 1. Profile of study enrollment. *Forty-nine patients were not enrolled because of initial misjudgment as non-aspiration pneumonia (7), initial misclassification of severity (4), i.v. or oral corticosteroid usage (2), protocol violation by physician (12), lack of informed consent (22), suspected concomitant infection of another organ (2).

Fig. 2. Clinical effective rate at the end of treatment for validated per-protocol population in TAZ/PIPC and IPM/CS groups.

Fig. 3. Time-course of axillary temperature (A), CRP (B), and WBC count (C) in modified intention-to-treatment (MITT) population. ${ }^{*} p<0.01$ compared to day 1 (Student's $t$-test); $\uparrow p<0.05$ in comparison between TAZ/PIPC group and IPM/CS group on day 4 (Mann-Whitney U test).

Fig. 4. Time-course of axillary temperature (A) and WBC count (B) in MITT population with gram-positive bacterial infection including mixed infection. ${ }^{*} p<0.001$, $* * p<0.01$ and $* * * p<0.05$ compared to day 1 (Student's t-test or Wilcoxon signed-rank test); $\uparrow p<0.01$ and $\uparrow \dagger p<0.05$ in comparison between TAZ/PIPC group and IPM/CS group on day4 (Student's t-test or Mann-Whitney U test). 
Table 1. Baseline clinical characteristics of modified intention-to-treat (MITT) population.

\begin{tabular}{|c|c|c|c|}
\hline & $\begin{array}{c}\text { TAZ/PIPC } \\
(\mathrm{n}=81)\end{array}$ & $\begin{array}{c}\mathrm{IPM} / \mathrm{CS} \\
(\mathrm{n}=82)\end{array}$ & p-value \\
\hline Male/female, n (\% male) & $37 / 44(46)$ & $47 / 35(57)$ & $0.14^{\mathrm{a}}$ \\
\hline Age, years & $84.6 \pm 7.0$ & $85.0 \pm 7.2$ & 0.67 \\
\hline NHAP, n (\%) & $32(40)$ & $44(54)$ & $0.07 \mathrm{a}$ \\
\hline Severity score (PSI) & $121 \pm 25$ & $128 \pm 26$ & 0.07 \\
\hline Class IV, n (\%) & $57(70)$ & $53(65)$ & $0.43^{\mathrm{a}}$ \\
\hline Class V, n (\%) & $24(30)$ & $29(35)$ & \\
\hline \multicolumn{4}{|l|}{ Performance status, n (\%) } \\
\hline 0 & $11(14)$ & $13(16)$ & $0.50^{\mathrm{a}}$ \\
\hline 1 & $17(21)$ & $7(9)$ & \\
\hline 2 & $18(22)$ & $20(24)$ & \\
\hline 3 & $13(16)$ & $18(22)$ & \\
\hline 4 & $22(27)$ & $24(29)$ & \\
\hline \multicolumn{4}{|l|}{ Maximum body temperature, ${ }^{\circ} \mathrm{C}$} \\
\hline Before treatment & $38.3 \pm 0.9$ & $38.3 \pm 0.9$ & 0.99 \\
\hline On the day of visit & $37.9 \pm 0.8$ & $37.9 \pm 0.8$ & 0.77 \\
\hline Systolic blood pressure, $\mathrm{mmHg}$ & $131.7 \pm 25.8$ & $128.9 \pm 28.0$ & 0.51 \\
\hline Diastolic blood pressure, $\mathrm{mmHg}$ & $74.8 \pm 15.6$ & $73.3 \pm 15.4$ & 0.53 \\
\hline Pulse rate, $\min ^{-1}$ & $91.3 \pm 17.3$ & $91.5 \pm 18.3$ & 0.92 \\
\hline Respiration rate, min $^{-1}$ & $24.1 \pm 6.1$ & $25.0 \pm 8.2$ & 0.48 \\
\hline $\mathrm{CRP}, \mathrm{mg} / \mathrm{dL}$ & $11.4 \pm 8.7$ & $11.2 \pm 9.4$ & 0.93 \\
\hline $\mathrm{WBC}, 1000 / \mu \mathrm{L}^{\mathrm{b}}$ & $10.1(2.3-21.3)$ & $10.2(4.7-43.2)$ & $0.23^{\mathrm{c}}$ \\
\hline Alb, mg/dL (cases) & $3.3 \pm 0.6(80)$ & $3.2 \pm 0.6(81)$ & 0.59 \\
\hline
\end{tabular}

TAZ/PIPC = tazobactam/piperacillin; IPM/CS = imipenem/cilastatin; $\mathrm{NHAP}=$ nursing-home-acquired pneumonia; $\mathrm{CRP}=\mathrm{C}$-reactive protein; $\mathrm{WBC}=$ white blood cells. Values are mean \pm SD unless otherwise indicated. Comparisons are made by Student's $t$-test unless otherwise indicated. aChi-square test, bMedian (range), cMann-Whitney $U$ test. 
Table 2. Clinical outcomes in the modified intention-to-treatment (MITT) and validated per-protocol (VPP) population

\begin{tabular}{lccc}
\hline & TAZ/PIPC & IPM/CS & p-value \\
\hline MITT population, n & 81 & 82 & \\
Treatment period, day & $10.6 \pm 4.2$ & $11.1 \pm 4.6$ & $0.48^{\mathrm{b}}$ \\
EOT, effective, n (\%) & $65 / 81(80)$ & $65 / 82(79)$ & 0.88 \\
EOS, cure, n (\%) & $49 / 81(60)$ & $52 / 82(63)$ & 0.63 \\
EOS, relapse, n (\%) & $7 / 81(9)$ & $10 / 82(12)$ & 0.45 \\
EOS, failure, n (\%) & $25 / 81(31)$ & $20 / 82(24)$ & 0.36 \\
Death within 30 days & $12 / 81(15)$ & $20 / 82(24)$ & 0.12 \\
VPP population, n & 76 & 79 & \\
Day 4, effective, n (\%) & $73 / 76(96)$ & $73 / 79(92)$ & 0.33 \\
Day 7, effective, n (\%) & $67 / 76(88)$ & $71 / 79(90)$ & 0.73 \\
EOT, effective, n (\%) & $63 / 76(83)$ & $65 / 79(82)$ & 0.92 \\
EOS, cure, n (\%) & $47 / 76(62)$ & $51 / 79(65)$ & 0.73 \\
EOS, relapse, n (\%) & $24 / 76(32)$ & $20 / 79(25)$ & 0.39 \\
EOS, failure, n (\%) & $5 / 76(7)$ & $8 / 79(10)$ & 0.43 \\
\hline
\end{tabular}

TAZ/PIPC = tazobactam/piperacillin; IPM/CS = imipenem/cilastatin; $\mathrm{EOT}=$ end of treatment; EOS = end of study. Relapse was defined as cases with re-fever after day 7 after primarily judged as effective by day 7 . Comparisons are made by chi-square test unless otherwise indicated. aMean $\pm \mathrm{SD}$, bStudent's $t$ test. 
Table 3. Presumptive causative pathogens in the two treatment groups

\begin{tabular}{lcc}
\hline & TAZ/PIPC & IPM/CS \\
\hline Streptococcus pneumoniae & $23(4)$ & $19(5)$ \\
MSSA & 0 & $3(1)$ \\
MRSA & 2 & $3(1)$ \\
Klebsiella pneumoniae & $4(2)$ & $2(2)$ \\
Escherichia coli & $3(1)$ & $3(1)$ \\
Haemophilus influenzae & $1(1)$ & $1(1)$ \\
Pseudomonas aeruginosa & $2(1)$ & $1(1)$ \\
Moraxella catarrhalis & 0 & 1 \\
Legionella pneumophila & 0 & 2 \\
Mycoplasma pneumoniae & $5(1)$ & $5(4)$ \\
Chlamydophila pneumoniae & 1 & $3(1)$ \\
Virus & 0 & 2 \\
Others & 2 & $8(4)$ \\
Unknown & 41 & 39 \\
\hline
\end{tabular}

MSSA = Methicillin-sensitive Staphylococcus aureus; MRSA = Methicillin-resistant

Staphylococcus aureus. Number of cases with other pathogens detected is indicated in parenthesis. 
Table 4. Clinical outcomes in the modified intention-to-treatment (MITT) and validated per-protocol (VPP) population stratified by presumptive causative bacteria

\begin{tabular}{|c|c|c|c|c|c|c|c|}
\hline & & \multicolumn{3}{|c|}{ Gram-positive cocci } & \multicolumn{3}{|c|}{ Gram-negative bacteria ${ }^{a}$} \\
\hline & & TAZ/PIPC & IPM/CS & $p$-value & TAZ/PIPC & $\mathrm{IPM} / \mathrm{CS}$ & p-value \\
\hline \multicolumn{2}{|c|}{ Cases with/without mixed infection, $\mathrm{n}$} & 26 & 31 & & 10 & 10 & \\
\hline \multirow[t]{2}{*}{ MITT } & Effective, EOT, n (\%) & $23 / 26(89)$ & $22 / 31(71)$ & 0.11 & $9 / 10(90)$ & $9 / 10(90)$ & 1 \\
\hline & Death, D30, n (\%) & $4 / 26(15)$ & $7 / 31(23)$ & 0.49 & $1 / 10(10)$ & $1 / 10(10)$ & 1 \\
\hline \multirow[t]{2}{*}{ VPP } & Effective, EOT, n (\%) & $22 / 23(96)$ & $22 / 30(73)$ & 0.032 & $1 / 10(10)$ & $0 / 9(0)$ & 0.32 \\
\hline & Death, D30, n (\%) & $3 / 23(13)$ & $6 / 30(20)$ & 0.50 & $1 / 10(10)$ & $1 / 10(10)$ & 1 \\
\hline \multicolumn{2}{|c|}{ Cases without mixed infection, n (\%) } & 22 & 22 & & 7 & 3 & \\
\hline MITT & Effective, EOT, n (\%) & $20 / 22(91)$ & $15 / 22(68)$ & 0.062 & 6/7 (86) & $3 / 3(100)$ & 0.49 \\
\hline VPP & Effective, EOT, n (\%) & $19 / 20(95)$ & $15 / 21(71)$ & 0.044 & 6/7 (86) & $3 / 3(100)$ & 0.49 \\
\hline
\end{tabular}

TAZ/PIPC = tazobactam/piperacillin; IPM/CS = imipenem/cilastatin $;$ EOT = end of treatment; MITT = modified intention-to-treatment;

VPP = validated per-protocol. aCases with Legionella infection were excluded. 
Table 5. Clinical outcomes in the modified intention-to-treatment (MITT) and validated per-protocol (VPP) population in patients without causative pathogens identified.

\begin{tabular}{llccc}
\hline & & TAZ/PIPC & IPM/CS & $\mathrm{p}$ \\
\hline \multirow{2}{*}{ MITT } & Effective, EOT, n (\%) & $31 / 41(76)$ & $32 / 39(82)$ & 0.48 \\
& Death, D30, n (\%) & $3 / 41(7)$ & $5 / 39(13)$ & 0.41 \\
\multirow{2}{*}{ VPP } & Effective, EOT, n (\%) & $30 / 39(77)$ & $32 / 37(86)$ & 0.28 \\
& Death, D30, n (\%) & $2 / 39(5)$ & $4 / 37(11)$ & 0.35 \\
\hline
\end{tabular}

Abbreviations are the same as Table 4. 
Table 6. Adverse events possibly or probably related to the study drug

\begin{tabular}{lcc}
\hline & TAZ/PIPC & IPM/CS \\
\hline Diarrhea (CD toxin positive) & $21(1)$ & $25(3)$ \\
Tarry stool & 0 & 1 \\
Seizure & 0 & 2 \\
Rash & 1 & 0 \\
Elevated AST or ALT & 1 & 3 \\
Acute renal failure & 0 & 1 \\
Thrombocytopenia & 1 & 0 \\
\hline Total & $24(24$ patients $)$ & $32(30$ patients $)$ \\
\hline
\end{tabular}

TAZ/PIPC = tazobactam/piperacillin; IPM/CS = imipenem/cilastatin. 


\section{References}

[1] United Nations Population Division. World population prospects: the 2006 revision. New York: United Nations Population Division; 2007.

[2] World Health Organization. The World Health Report 2004. Geneva: World Health Organization; 2004.

[3] Ministry of Health Labour and Welfare, Government of Japan. Summary of vital statistics 2006 (in Japanese). Tokyo: Ministry of Health Labour and Welfare, Government of Japan; 2007.

[4] Kikuchi R, Watabe N, Konno T, Mishina N, Sekizawa K, Sasaki H. High incidence of silent aspiration in elderly patients with community-acquired pneumonia. Am J Respir Crit Care Med 1994; 150:251-3.

[5] Hammond JM, Potgieter PD, Hanslo D, Scott H, Roditi D. The etiology and antimicrobial susceptibility patterns of microorganisms in acute community-acquired lung abscess. Chest 1995; 108:937-41.

[6] Wang JL, Chen KY, Fang CT, Hsueh PR, Yang PC, Chang SC. Changing bacteriology of adult community-acquired lung abscess in Taiwan: Klebsiella pneumoniae versus anaerobes. Clin Infect Dis 2005; 40:915-22.

[7] Bartlett JG, Gorbach SL, Finegold SM. The bacteriology of aspiration pneumonia. Am J Med 1974; 56:202-7.

[8] El-Solh AA, Pietrantoni C, Bhat A, Aquilina AT, Okada M, Grover V, et al. Microbiology of severe aspiration pneumonia in institutionalized elderly. Am J Respir Crit Care Med 2003; 167:1650-4.

[9] Sanguinetti CM, De Benedettob F, Miragliotta G, DEDALO Study Group. Bacterial agents of lower respiratory tract infections (LRTIs), $\beta$-lactamase production, 
and resistance to antibiotics in elderly people. Int J Antimicrob Agents 2000; $16: 467-71$.

[10] Templeton KE, Scheltinga SA, van den Eeden WC, Graffelman AW, van den Broek PJ, Claas EC. Improved diagnosis of the etiology of community-acquired pneumonia with real-time polymerase chain reaction. Clin Infect Dis 2005; 41:345-51.

[11] Kadowaki M, Demura Y, Mizuno S, Uesaka D, Ameshima S, Miyamori I, et al. Reappraisal of clindamycin IV monotherapy for treatment of mild-to-moderate aspiration pneumonia in elderly patients. Chest 2005; 127:1276-82.

[12] Tokuyasu H, Harada T, Watanabe E, Okazaki R, Touge H, Kawasaki Y, et al. Effectiveness of meropenem for the treatment of aspiration pneumonia in elderly patients. Intern Med 2009; 48:129-35.

[13] Yanagihara K, Fukuda Y, Seki M, Izumikawa K, Higashiyama Y, Miyazaki Y, et al. Clinical comparative study of sulbactam/ampicillin and imipenem/cilastatin in elderly patients with community-acquired pneumonia. Intern Med 2006; 45:995-9. [14] Carmeli Y, Troillet N, Eliopoulos GM, Samore MH. Emergence of antibiotic-resistant Pseudomonas aeruginosa: comparison of risks associated with different antipseudomonal agents. Antimicrob Agents Chemother 1999; 43:1379-82. [15] Lepper PM, Grusa E, Reichl H, Högel J, Trautmann M. Consumption of imipenem correlates with ß-lactam resistance in Pseudomonas aeruginosa. Antimicrob Agents Chemother 2002; 46:2920-5.

[16] Mandell LA, Wunderink RG, Anzueto A, Bartlett JG, Campbell GD, Dean NC, et al. Infectious diseases society of America/American thoracic society consensus guidelines on the management of community-acquired pneumonia in adults. Clin Infect Dis 2007; 44:S27-S72. 
[17] Fine MJ, Auble TE, Yealy DM, Hanusa BH, Weissfeld LA, Singer DE, et al. A prediction rule to identify low-risk patients with community-acquired pneumonia. $\mathrm{N}$ Engl J Med 1997; 336:243-50.

[18] British Thoracic Society Pneumonia Guidelines Committee. BTS guidelines for the management of community acquired pneumonia in adults-2004 update.

[19] Ott SR, Allewelt M, Lorenz J, Reimnitz P, Lode H, German Lung Abscess Study Group. Moxifloxacin vs ampicillin/sulbactam in aspiration pneumonia and primary lung abscess. Infection 2008; 36:23-30.

[20] Allewelt M, Schüler P, Bölcskei PL, Mauch H, Lode H, Study Group on Aspiration Pneumonia. Ampicillin + sulbactam vs. clindamycin \pm cephalosporin for the treatment of aspiration pneumonia and primary lung abscess. Clin Microbiol Infect 2004; 10:163-70.

[21] Marik PE. Aspiration pneumonitis and aspiration pneumonia. N Engl J Med 2001; 344:665-71.

[22] Ito I, Ishida T, Togashi K, Niimi A, Koyama H, Ishimori T, et al. Differentiation of bacterial and non-bacterial community-acquired pneumonia by thin-section computed tomography. Eur J Radiol 2009; 72:388-95.

[23] Hoellman DB, Kelly LM, Credito K, Anthony L, Ednie LM, Jacobs MR, et al. In vitro antianaerobic activity of ertapenem (MK-0826) compared to seven other compounds. Antimicrob Agents Chemother 2002; 46:220-4.

[24] Edmiston CE Jr, Krepel CJ, Kehl KS, Seabrook GR, Somberg LB, Almassi GH, et al. Comparative in vitro antimicrobial activity of a novel quinolone, garenoxacin, against aerobic and anaerobic microbial isolates recovered from general, vascular, cardiothoracic and otolaryngologic surgical patients. J Antimicrob Chemother 2005; 
$56: 872-8$.

[25] Cometta A, Zinner S, de Bock R, Calandra T, Gaya H, Klastersky J, et al. Piperacillin-tazobactam plus amikacin versus ceftazidime plus amikacin as empiric therapy for fever in granulocytopenic patients with cancer. The International Antimicrobial Therapy Cooperative Group of the European Organization for Research and Treatment of Cancer. Antimicrob Agents Chemother 1995; 39:445-52. [26] Maruyama T, Niederman MS, Kobayashi T, Kobayashi H, Takagi T, D'Alessandro-Gabazza CN, et al. A prospective comparison of nursing home-acquired pneumonia with hospital-acquired pneumonia in non-intubated elderly. Respir Med 2008; 102:1287-95.

[27] Shindo Y, Sato S, Maruyama E, Ohashi T, Ogawa M, Hashimoto N, et al. Health-care-associated pneumonia among hospitalized patients in a Japanese community hospital. Chest 2009; 135:633-40.

[28] Micek ST, Kollef KE, Reichley RM, Roubinian N, Kollef MH. Health care-associated pneumonia and community-acquired pneumonia: a single-center experience. Antimicrob Agents Chemother 2007; 51:3568-73.

[29] Kollef MH, Shorr A, Tabak YP, Gupta V, Liu LZ, Johannes RS. Epidemiology and outcomes of health-care-associated pneumonia. Results from a large US database of culture-positive pneumonia. Chest 2005; 128:3854-62.

[30] American Thoracic Society; Infectious Diseases Society of America. Guidelines for the management of adults with hospital-acquired, ventilator-associated, and healthcare-associated pneumonia. Am J Respir Crit Care Med 2005; 171:388-416. [31] Jaccard C, Troillet N, Harbarth S, Zanetti G, Aymon D, Schneider R, et al. $\underline{\text { Prospective randomized comparison of imipenem-cilastatin and piperacillin-tazobactam }}$ 
in nosocomial pneumonia or peritonitis. Antimicrob Agents Chemother 1998;

42:2966-72.

[32] Joshi M, Metzler M, McCarthy M, Olvey S, Kassira W, Cooper A. Comparison of piperacillin/tazobactam and imipenem/cilastatin, both in combination with tobramycin, administered every $6 \mathrm{~h}$ for treatment of nosocomial pneumonia. Respir Med. 2006;100:1554-65.

[33] Schmitt DV, Leitner E, Welte T, Lode H. Piperacillin/tazobactam vs imipenem/vilastatin in the treatment of nosocomial pneumonia-a double blind prospective multicentre study. Infection 2006; 34:127-34.

[34] Joshi M, Bernstein J, Solomkin J, Wester BA, Kuye O. Piperacillin/tazobactam plus tobramycin versus ceftazidime plus tobramycin for the treatment of patients with $\underline{\text { nosocomial lower respiratory tract infection. J Antimicrob Chemother 1999; 43:389-97. }}$ [35] Brun-Buisson C, Sollet JP, Schweich H, Brière S, Petit C. Treatment of ventilator-associated pneumonia with piperacillin-tazobactam/amikacin versus ceftazidime/amikacin: a multicenter, randomized controlled trial. Clin Infect Dis 1998; $\underline{26: 346-54 .}$

[36] Matsumoto K, Nagatake T, Oishi K, Amamoto T, Urae R, Niki Y, et al. Evaluation of emergence of beta-lactam resistance in Pseudomonas aeruginosa (in Japanese). Chemotherapy 1994; 42(S2): 281-99

[37] Mikamo H, Yamagishi Y. Strategic use of antimicrobial agents based on pharmacokinetics-pharmacodynamics (PK-PD) theory (in Japanese). ICC \& CCU 2008; 32: 269-79.

[38] Kim MK, Capitano B, Mattoes HM, Xuan D, Quintiliani R, Nightingale CH, et al. Pharmacokinetic and pharmacodynamic evaluation of two dosing regimens for 
piperacillin-tazobactam. Pharmacotherapy 2002; 5:569-77.

[39] Occhipinti DJ, Pendland SL, Schoonover LL, Rypins EB, Danziger LH, Rodvold KA. Pharmacokinetics and pharmacodynamics of two multiple-dose piperacillin-tazobactam regimens. Antimicrob Agents Chemother 1997; 41:2511-7. [40] Frei CR, Burgess DS. Pharmacokinetic/pharmacodynamic modeling to predict in vivo effectiveness of various dosing regimens of piperacillin/tazobactam and piperacillin monotherapy against gram-negative pulmonary isolates from patients managed in intensive care units in 2002. Clin Ther 2008; 30:2335-41.

[41] Higashiya F, Mihashi S. Evaluation of antimicrobial effects and optimal ratio of tazobactam/piperacillin combination (in Japanese). Chemotherapy 1994; 42(S2): 26-33 


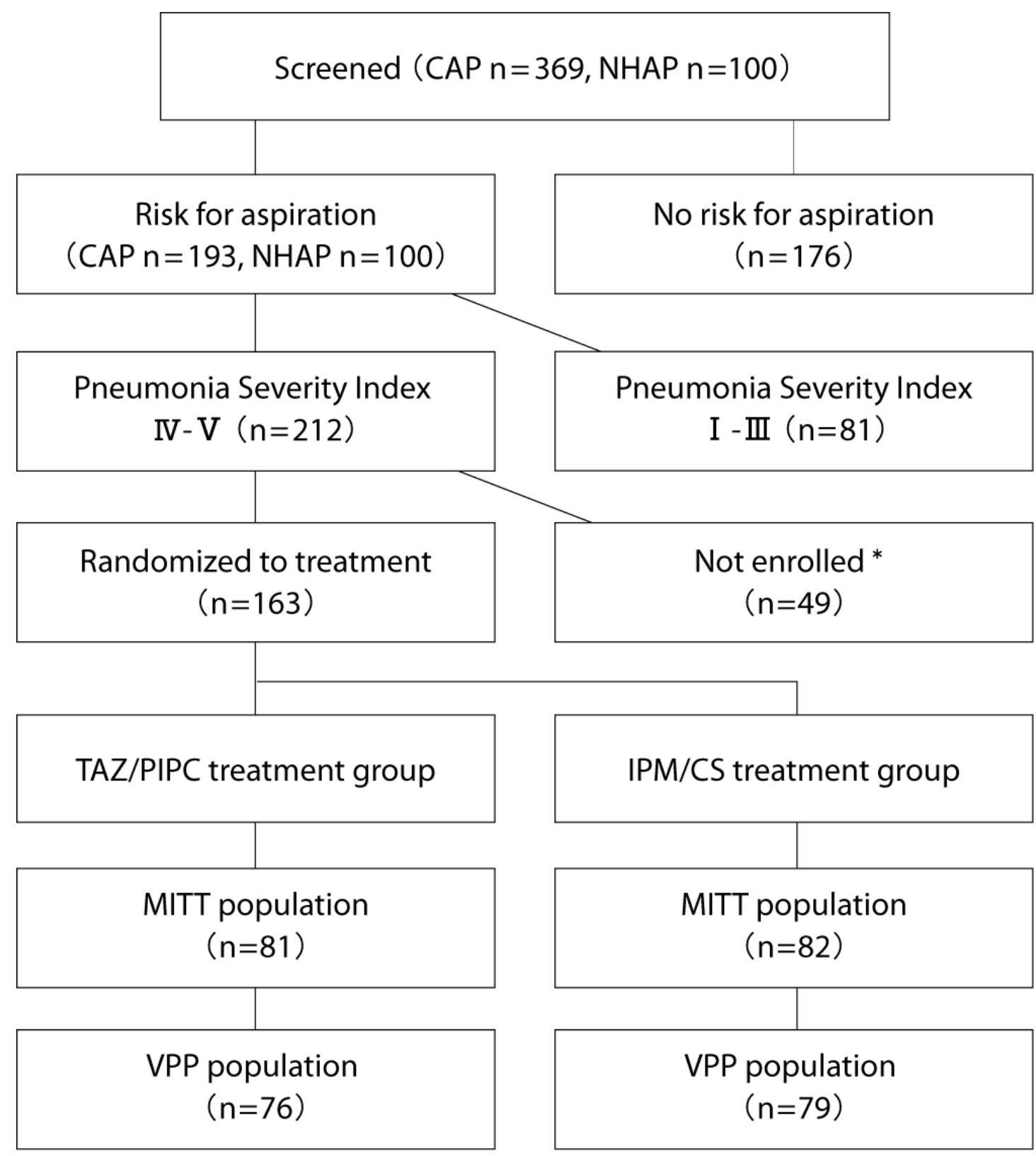

Figure 1 
NS

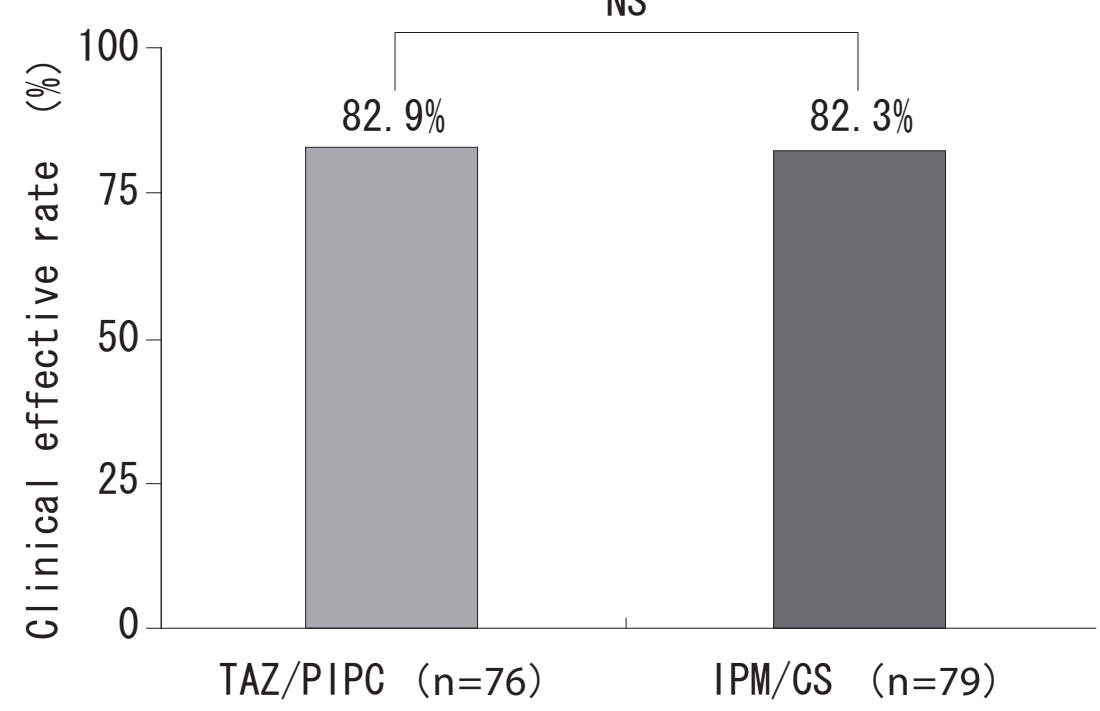

Figure 2 
A) Axillary temperature

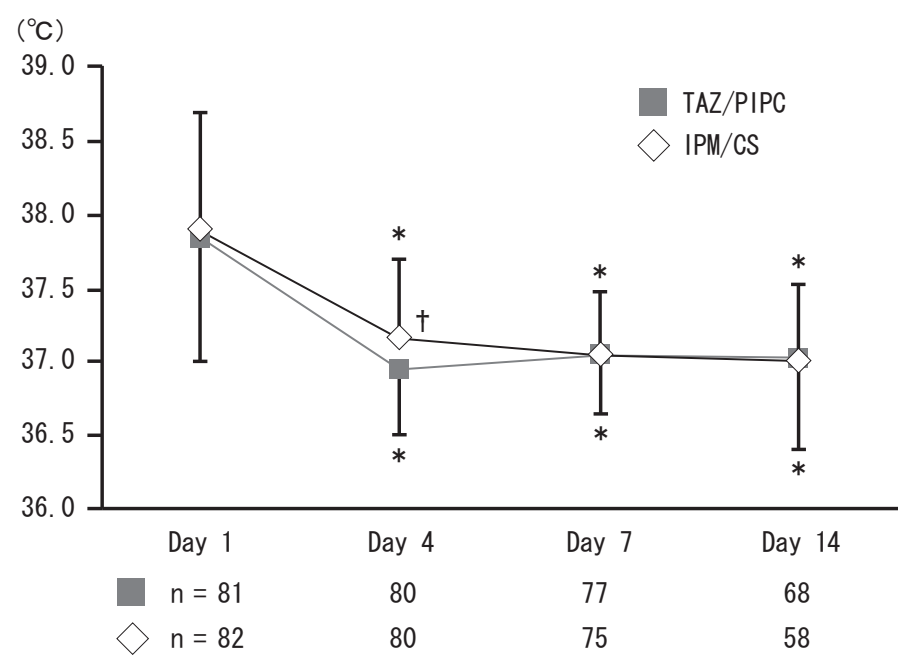

C) WBC

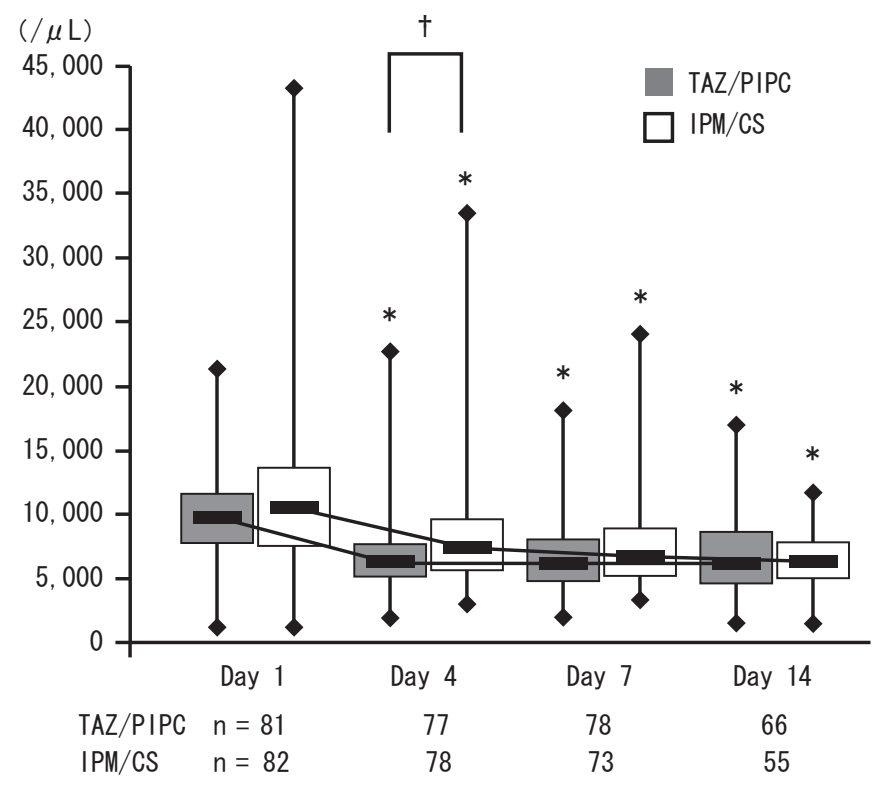

B) CRP

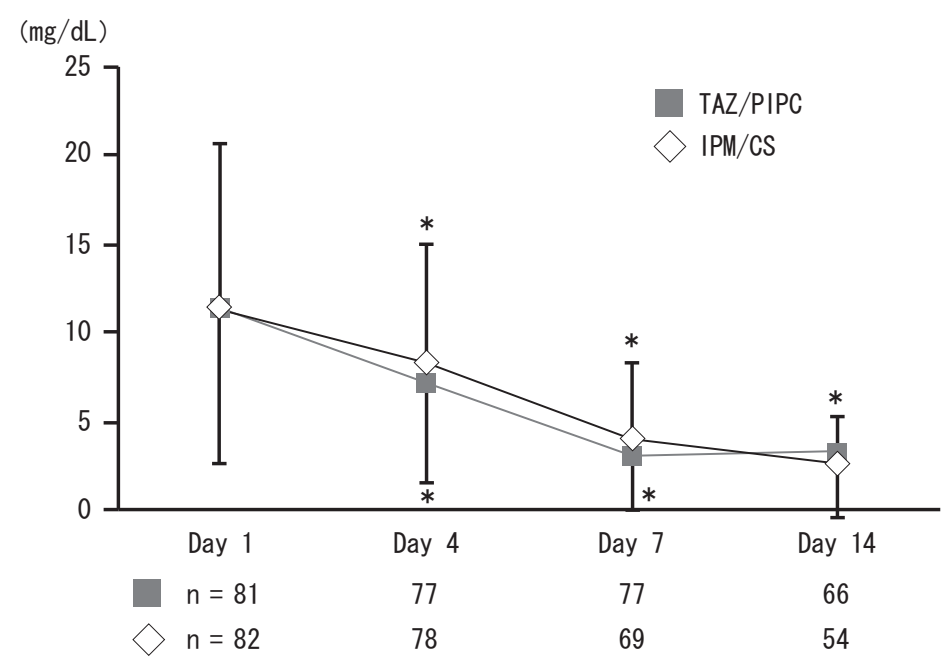

Figure 3 
A) Axillary temperature

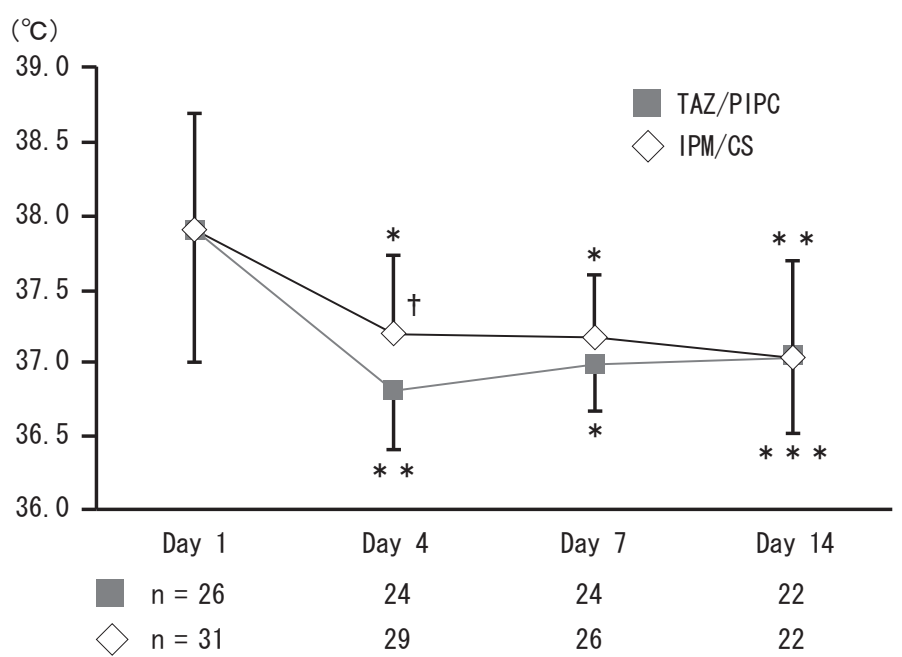

B) WBC

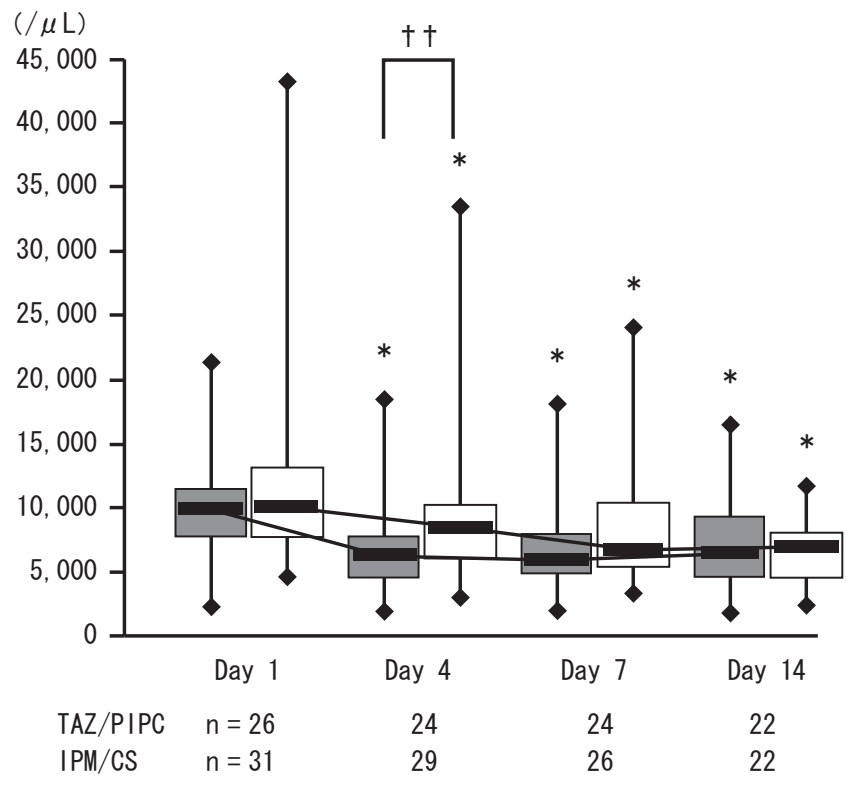

Figure 4 